\title{
Are pangenomes adaptive or not?
}

To the Editor - Most types of bacteria can rapidly take up and lose genes, resulting in different strains carrying unique selections of accessory genes drawn from the species' 'pangenome'. It is well established that acquiring accessory genes can have adaptive, neutral or deleterious effects on fitness, but the relative prevalence of these different types of changes remains an important open question in evolutionary microbiology ${ }^{1}$.

In a recent Perspective in Nature Microbiology, McInerney et $\mathrm{al}^{2}$ argue that accessory genes mostly have beneficial effects on fitness. Although we cannot disprove this scenario, we find that their arguments are inconclusive. They argue that it is unlikely that most gene content changes are deleterious. First, based on a comparative genomics analysis, they suggest that selfish genetic elements (which are most likely to be deleterious) are not the dominant class of gene content changes. However, at least two other studies have found that selfish elements do dominate the accessory genome $^{3,4}$. Furthermore, this in itself is not an argument that any of the more commonly found types of gene content changes would not be deleterious. Second, the authors argue that the mere observation of high genome flexibility points at its adaptiveness, otherwise it would not be found. However, this is not the case when selection to lower the uptake of accessory genes that are generally deleterious is outweighed by the occasional uptake of adaptive genes, other (for example, nutritional) benefits, or when selection against the influx of genes is constrained or not even possible ${ }^{5}$. Likewise, they argue that if most accessory genes are predominantly neutral or slightly deleterious then this would result in the expansion of genomes, which is not observed. However, this assumes that the rate of deletion is independent of genome size, which might be unlikely as expanding genomes can be expected to become gradually more unstable, favouring larger deletions. It also assumes that lateral gene transfer processes primarily result in gene gain and not gene loss, and this might not always be the case ${ }^{6}$.

McInerney et al. make the case for the general advantageousness of accessory genes based on three lines of evidence. The empirical evidence does not convince us one study cited by McInerney et al. found that experimental deletion of accessory genes is mostly deleterious, suggesting that the presence of these genes is mostly advantageous. However, in this study ${ }^{7}$, every deletion involved multiple genes, including core genes. This makes these results inconclusive, as deletion of beneficial genes could have been accompanied by deletion of multiple neutral or slightly deleterious genes. On a theoretical note, they argue that species with large effective population size $\left(N_{\mathrm{e}}\right)$ have large pangenomes, as these species experience more effective selection. Although it is true that species with larger $N_{\mathrm{e}}$ are expected to have a lower proportion of deleterious polymorphism, they still are expected to carry a higher absolute number of such non-adaptive changes. The argument made by McInerney et al. for the general adaptiveness of the observation of many rare, short-lived accessory genes mainly rests on the assumption that different strains, sharing few accessory genes, each inhabit distinct, transient niches. Pioneering work by Golding and colleagues ${ }^{8}$ has shown that genes are gained and lost at higher rates on the tips of the phylogenetic tree when the tree is constructed using synonymous variation. This pattern could fit the scenario given by McInerney et al., where genes are transiently advantageous in only a small subset of strains. However, we see no reason to abandon the more parsimonious explanation that most accessory genes are rare just because they have (slightly) deleterious effects on fitness. The recent result by Andreani et al. ${ }^{9}$ is consistent with such a nearly neutral scenario.

As bacteria cannot select which particular genes they take up, we expect most gene content changes to not be adaptive. However, it is important to note that gene transfer processes can be generally advantageous even when the majority of gene content changes are (slightly) deleterious, or that they can be deleterious even when occasionally giving rise to adaptations. It is difficult to think of data that will resolve the question of whether accessory genomes are dominated by deleterious, neutral or adaptive genetic variation. Purely sequence-based analysis is hampered by the fact that gene content changes cannot be divided into classes with different selective expectations, as is the case for nucleotide variation (that is, synonymous and non-synonymous polymorphism). Experiments combining careful genome editing to add or delete accessory genes could be useful, but are contingent on being able to perform sensitive fitness assays in relevant environments. By whatever approach, we hope that researchers take up the gauntlet to provide new data that could help resolve this important evolutionary puzzle.

Michiel Vos ${ }^{1 *}$ and Adam Eyre-Walker ${ }^{2 *}$ ${ }^{1}$ European Centre for Environment and Human Health, University of Exeter Medical School, University of Exeter, Penryn, UK. ${ }^{2}$ School of Life Sciences, University of Sussex, Falmer, UK.

*e-mail:m.vos@exeter.ac.uk; a.c.eyre-walker@sussex.ac.uk

Published online: 24 November 2017 https://doi.org/10.1038/s41564-017-0067-5

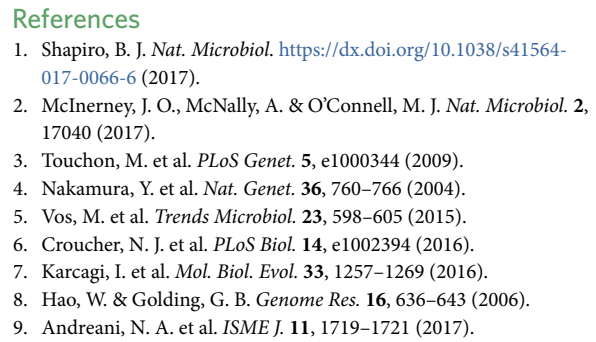

Competing interests

The authors declare no competing financial interests. 